\title{
An Approach to Interval Fuzzy Probabilities
}

\author{
Tiago da Cruz Asmus ${ }^{1}$ Graçaliz Pereira Dimuro ${ }^{2}$ Benjamín Bedregal $^{3}$ \\ ${ }^{1}$ IMEF, Universidade Federal do Rio Grande, Rio Grande, Brazil \\ ${ }^{2}$ PPGCOMP, C3, Universidade Federal do Rio Grande, Rio Grande, Brazil \\ ${ }^{3}$ DIMAP, Universidade Federal do Rio Grande do Norte, Natal, Brazil
}

\begin{abstract}
Fuzzy sets and logic have been largely used for the treatment of the uncertainty, vagueness and ambiguity found in the modeling of real problems. However, there may exist also the case when there is uncertainty related to the membership functions to be used in the modeling of fuzzy sets and fuzzy numbers, as there are many ways to define the shape of this kind of number. Thus, one can use, for example, the theory of interval fuzzy sets to address this uncertainty, considering different modelings of fuzzy numbers into a single interval fuzzy number. In this paper, we use interval fuzzy numbers to represent probabilities that are difficult to be estimated and where the modeling of fuzzy numbers is not trivial. To elaborate the calculation of interval fuzzy probabilities, we introduce an approach based on the one used by Buckley and Eslami for fuzzy probabilities, where the probabilities respect an arithmetic restriction. We discuss several properties of the proposed approach.
\end{abstract}

Keywords: interval fuzzy numbers, ranking interval fuzzy numbers, interval fuzzy probabilities, interval fuzzy mean

\section{Introduction}

In problems of decision making in an environment with uncertainty, the decision maker must estimate the probabilities of different actions, which may lead to different outcomes. However, sometimes these probabilities are difficult to estimate precisely, such as problems in agent-based social simulation $[1,2,3]$, which often have linguistic variables to define some parameters of the agents involved in the modeling $[4,5,6]$ under vagueness, ambiguity and uncertainty.

However, one can find in the theory of fuzzy sets an alternative to model this imprecision, as fuzzy numbers are ideal for representing linguistic variables and modeling imprecise values $[7,8]$. But still there may be uncertainty in how to model this fuzzy numbers, as there are many ways to define the shape of this kind of number. Thus, one can use the theory of interval fuzzy sets to address this uncertainty, considering different modelings of fuzzy numbers into a single interval fuzzy number [9].

In this paper, we use interval fuzzy numbers to represent probabilities that are difficult to be esti- mated and where the modeling of fuzzy numbers is not trivial. To elaborate the calculation of interval fuzzy probabilities, we introduce an approach based on the one used by Buckley and Eslami [10], where the probabilities respect an arithmetic restriction on the interval $[0,1]$. We discuss several properties of the proposed approach.

In the literature, we can find other approaches to fuzzy probabilities. For example, the approach proposed by da Costa [11], without the use of an arithmetic restriction, facilitates the calculation of each probability, avoiding the interdependence between them, but it produces fuzzy numbers with greater dispersion than the ones produced by our approach. As the probabilities defined in this paper are calculated to be utilized in problems of decision making, we prefer to work with fuzzy values with the least possible dispersion to avoid ambiguous cases in the ordering of imprecise values.

The paper is organized as follows. In Section 2, we provide the notion of interval fuzzy numbers, their arithmetic and a total order for symmetric triangular and positive interval fuzzy numbers. In Section 3, preliminary aspects and the aforementioned approach for fuzzy probabilities is presented, as the definition of fuzzy mean ${ }^{1}$. In Section 4 , the focus is on development of the approach to interval fuzzy probabilities, joint with some propositions and properties, and the definition of interval fuzzy mean. Section 5 brings the final considerations of this paper and some ideas for future work.

\section{Interval Fuzzy Numbers}

The fuzzy set theory is a useful tool for modeling uncertainty. However, sometimes it is difficult to determine the membership degree to be used for certain problems. To work around this situation, several authors (e.g., as discussed in $[14,15,16,17])$ represent the membership degrees through real intervals, thus extending fuzzy sets to interval fuzzy sets.

Considering $\mathbb{I R}$ as the set of all real intervals, let $U I=[0,1] \in \mathbb{I R}$ be the real unit interval, and define $\mathbb{U}=\{[a, b] \mid 0 \leq a \leq b \leq 1\}$ as the set of all sub-intervals of $U I$.

Thus, an interval fuzzy subset $A$ of a universe $\mathbb{X}$

\footnotetext{
${ }^{1}$ For the lack of space, we omit some proofs of propositions and properties, which can be found in $[12,13]$.
} 
is defined as the set of ordered pairs

$$
A=\left\{\left(x, \mu_{A}(x)\right) \mid x \in \mathbb{X}\right\}
$$

where $\mu_{A}: \mathbb{R} \rightarrow \mathbb{U}$ is the interval membership function of $A$.

If the interval membership function $\mu_{A}$ is continuous $^{2}$, then there are continuous functions $\mu_{A_{l}}, \mu_{A_{u}}$ : $\mathbb{X} \rightarrow U I$ called respectively as lower membership function (LMF) and upper membership function (UMF), such that, for every $x \in \mathbb{X}$ :

$$
\mu_{A}(x)=\left[\mu_{A_{l}}(x), \mu_{A_{u}}(x)\right]
$$

where $\mu_{A_{l}}(x) \leq \mu_{A_{u}}(x)$. The inner and outer supports of an interval fuzzy set $A$ of $\mathbb{X}$ are defined, respectively, by:

$$
\begin{aligned}
\operatorname{lsupp}_{A} & =\left\{x \in \mathbb{X} \mid \mu_{A_{l}}(x)>0\right\} \\
\operatorname{usupp}_{A} & =\left\{x \in \mathbb{X} \mid \mu_{A_{u}}(x)>0\right\} .
\end{aligned}
$$

For the same $A$, the core of this interval fuzzy subset is defined by:

$$
\text { core }_{A}=\left\{x \in \mathbb{X} \mid \mu_{A}(x)=[1 ; 1]\right\} .
$$

In other words:

$$
\text { core }_{A}=\left\{x \in \mathbb{X} \mid \mu_{A_{l}}(x)=\mu_{A_{u}}(x)=1\right\} .
$$

For $\alpha=\left[\alpha_{1}, \alpha_{2}\right] \in \mathbb{U}$, we define the $\left[\alpha_{1}, \alpha_{2}\right]$-cuts of $A$ as:

$A\left[\alpha_{1}, \alpha_{2}\right]= \begin{cases}\left\{x \in \mathbb{X} \mid \mu_{A}(x) \geq_{K} \alpha\right\}, & \text { if } \alpha_{1} \neq 0 \\ A_{l}[0] \cap\left\{x \in \mathbb{X} \mid \mu_{A}(x) \geq_{K} \alpha\right\}, & \text { if } \alpha_{1}=0 \neq \alpha_{2} \\ A_{l}[0] \cap A_{u}[0], & \text { if } \alpha_{1}=\alpha_{2}=0 .\end{cases}$

where $A_{l}[0]$ is the closure of the support of $A_{l}$ and $A_{u}[0]$ is the closure of the support of $A_{u}$. As in the classical fuzzy theory, an interval fuzzy set is completely determined by its $\left[\alpha_{1}, \alpha_{2}\right]$-cuts.

An interval fuzzy number is defined as an interval extension of the usual definition of the fuzzy number, considering the approach of interval fuzzy sets presented here. Therefore, an interval fuzzy number $\hat{N}$ is defined as a interval fuzzy set of $\mathbb{R}$ with the following characteristics: [9]

(a) the $\left[\alpha_{1}, \alpha_{2}\right]$-cuts and the core of $\hat{N}$ are real intervals, that is, $\hat{N}\left[\alpha_{1}, \alpha_{2}\right]$, core $_{\hat{N}} \in \mathbb{I R}$;

(b) $\operatorname{lsupp}_{\hat{N}}$ and $u s u p p_{\hat{N}}$ are bounded.

If the functions LMF and UMF are both linear, then $\hat{N}$ is called linear interval fuzzy number, which can be defined by an interval membership function $\mu_{\hat{N}}$, with supports $l \operatorname{supp}_{\hat{N}}=\left(a_{l}, b_{l}\right)$,

\footnotetext{
${ }^{2}$ The continuity of interval functions was defined by Moore as an extension of the continuity of real functions. More information on this subject can be seen in $[18,19,20]$.
}

usupp $_{\hat{N}}=\left(a_{u}, b_{u}\right)$ and core core $_{\hat{N}}=\left[u_{1}, u_{2}\right]$, and is denoted by the tuple

$$
\left(\left[a_{u}, a_{l}\right] /\left[u_{1}, u_{2}\right] /\left[b_{l}, b_{u}\right]\right) \text {. }
$$

If $u_{1}=u_{2}=u$, then $\hat{N}$ is a triangular (linear) interval fuzzy number. The set of all interval fuzzy numbers is denoted by $\hat{\mathbb{F}}(\mathbb{R})$.

One can observe that the functions LMF and UMF describe, respectively, the fuzzy numbers $\bar{N}_{l}$ e $\bar{N}_{u}$, which can represent the interval fuzzy number $\hat{N}$. Assuming that $\bar{N}_{l}$ and $\bar{N}_{u}$ are both symmetric triangular fuzzy numbers described respectively by the functions $\mu_{\hat{N}_{i}}(\mathrm{LMF})$ and $\mu_{\hat{N}_{s}}(\mathrm{UMF})$, and represented respectively by the tuples $\left(a_{l} / u / b_{l}\right)$ and $\left(a_{u} / u / b_{u}\right)$, then $\hat{N}$ is a symmetric triangular interval fuzzy number.

Thus, an interval fuzzy number may be represented as an ordered pair of fuzzy numbers. Therefore, $\hat{N}=\left(\bar{N}_{l}, \bar{N}_{u}\right)$, where $\bar{N}_{l}$ and $\bar{N}_{u}$ are called lower generator number and upper generator number, respectively, as they constitute $\hat{N}$.

The addition of two triangular interval fuzzy numbers

$$
\hat{N}_{1}=\left(\left[a_{u}, a_{l}\right] /\left[u_{1}, u_{2}\right] /\left[b_{u}, b_{l}\right]\right)
$$

and

$$
\hat{N}_{2}=\left(\left[c_{u}, c_{l}\right] /\left[v_{1}, v_{2}\right] /\left[d_{u}, d_{l}\right]\right)
$$

is the triangular interval fuzzy number

$\hat{N}=\left(\left[a_{u}+c_{u}, a_{l}+c_{l}\right] /\left[u_{1}+v_{1}, u_{2}+v_{2}\right] /\left[b_{u}+d_{u}, b_{l}+d_{l}\right]\right)$.

Clearly, in this case, one has that

$$
\hat{N}=\left(\bar{N}_{1 l}+\bar{N}_{2 l}, \bar{N}_{1 u}+\bar{N}_{2 u}\right)
$$

where the addition is the usual adition among triangular fuzzy numbers. Analogously, the pseudoinverse additive of a triangular interval fuzzy number $\hat{N}=\left(\left[a_{u}, a_{l}\right] /\left[u_{1}, u_{2}\right] /\left[b_{u}, b_{l}\right]\right)$ is the triangular interval fuzzy number

$$
-\hat{N}=\left(\left[-b_{l},-b_{u}\right] /\left[-u_{2},-u_{1}\right] /\left[-a_{l},-a_{u}\right]\right)
$$

or equivalently, $-\hat{N}=\left(-\bar{N}_{u},-\bar{N}_{l}\right)$.

Based on the concept that two symmetric triangular fuzzy generator numbers with the same core produce a symmetric triangular interval fuzzy number, an order relation was defined, based on the $\mathrm{AD}$-order for comparing the fuzzy generator numbers, and analyzing those comparisons to determine the ordering of symmetric triangular interval fuzzy numbers. [21]

Let $\bar{F}_{1 l}$ and $\bar{F}_{1 u}$ be two symmetric triangular interval fuzzy numbers obtained through the functions LMF and UMF, respectively, of an interval fuzzy number $\hat{F}_{1}$. Analogously, let $\bar{F}_{2 l}$ e $\bar{F}_{2 u}$ be, respectively, the lower and upper fuzzy generator numbers of another symmetric triangular interval fuzzy number, $\hat{F}_{2}$. Then, the relation $\hat{<}$ is defined by: 


$$
\hat{F}_{1} \hat{<} \hat{F}_{2} \Leftrightarrow\left(\bar{F}_{1 u} \overline{<} \bar{F}_{2 u}\right) \vee\left(\bar{F}_{1 u}=\bar{F}_{2 u} \wedge \bar{F}_{1 l} \overline{<} \bar{F}_{2 l}\right)
$$

where $\overline{<}$ is given in the next Definition.

Definition 1 Given a fix $\rho \in[0,1], \bar{F}_{1} \overline{<} \bar{F}_{2}$ if and only if one of the following conditions hold:

(1) $\left(a_{1}<a_{2}\right) \wedge\left(b_{1} \leq b_{2}\right)$;

(2) $\left(a_{1}<a_{2}\right) \wedge\left(b_{2}<b_{1}\right) \wedge\left(u_{1} \leq u_{2}\right)$;

(3) $\left(a_{1}<a_{2}\right) \wedge\left(b_{2}<b_{1}\right) \wedge\left(u_{2}<u_{1}\right) \wedge$

$\left[\forall \alpha:(0 \leq \alpha \leq \rho) \Rightarrow\left(u_{1}-a_{1}\right) \alpha+a_{1} \leq\left(u_{2}-a_{2}\right) \alpha+a_{2}\right] ;$

(4) $\left(a_{2} \leq a_{1}\right) \wedge\left(b_{1}<b_{2}\right) \wedge\left(u_{1}<u_{2}\right)$

$$
\left[\exists \alpha:(0 \leq \alpha \leq \rho) \wedge\left(u_{1}-a_{1}\right) \alpha+a_{1}<\left(u_{2}-a_{2}\right) \alpha+a_{2}\right] .
$$

Then, one has that:

$$
\hat{F}_{1} \hat{\varkappa} \hat{F}_{2} \Leftrightarrow \hat{F}_{1}=\hat{F}_{2} \vee \hat{F}_{1} \hat{<} \hat{F}_{2} .
$$

This relation, as the AD-order, take the information precision contained in each number into consideration as a factor to be analyzed when interval fuzzy numbers are ordered. This relation also satisfies the properties of reflexivity, antisymmetry and transitivity, so it is an order relation. Finally, one has that:

$$
\forall \hat{F}_{1}, \hat{F}_{2}: \hat{F}_{1}=\hat{F}_{2} \underline{\vee} \hat{F}_{1} \hat{<} \hat{F}_{2} \underline{\vee} \hat{F}_{2} \hat{<} \hat{F}_{1}
$$

Therefore, the relation defined by the Equation (9) to be total. The proofs of these properties are presented in [21].

\section{Fuzzy Probabilities}

For the purpose of this paper, we consider triangular and symmetrical fuzzy numbers, denoted by

$$
\bar{F}=(a / u / b)
$$

with $u$ being the core of the fuzzy number, and its $\alpha$-cuts represented by

$$
\bar{F}[\alpha]=[(u-a) \alpha+a,(u-b) \alpha+b] .
$$

Interval fuzzy numbers [9] can be represented by pairs of generator fuzzy numbers as $\hat{F}=\left(\bar{F}_{i}, \bar{F}_{s}\right)$, where $\bar{F}_{i}$ and $\bar{F}_{s}$ are defined by the lower and upper membership functions of $\hat{F}$, respectively. The $\left[\alpha_{1}, \alpha_{2}\right]$-cuts of $\hat{F}$ are given by

$$
\hat{F}\left[\alpha_{1}, \alpha_{2}\right]=\bar{F}_{i}\left[\alpha_{1}\right] \cap \bar{F}_{s}\left[\alpha_{2}\right] .
$$

For ordering interval fuzzy numbers, we adopt the Interval AD-Order [21].

We present a general definition, through stochastic vectors, for the approach proposed by Buckley to calculate fuzzy probabilities [10, 22], which does not consider the standard probability theory [23]. Let $X=\left\{x_{1}, \ldots, x_{n}\right\}$ be a finite set and $P: \wp(X) \rightarrow$ $[0,1]$ a probability function defined for all subsets of $X$, with $P\left(\left\{x_{i}\right\}\right)=\phi_{i}, 1 \leq i \leq n, 0 \leq \phi_{i} \leq 1$ and $\sum_{i=1}^{n} \phi_{i}=1$. Thus, $(X, P)$ characterizes a finite and discrete probability distribution, represented by the set $\Phi=\left\{\phi_{1}, \ldots, \phi_{n}\right\}$.

We observe that the elements of $\Phi$ are often obtained by experts' opinions, not always representing precise values or consensus. To model this uncertainty, one may use fuzzy numbers $\bar{\phi}_{i}$, forming a new set denoted by $\bar{\Phi}$. Thus, the fuzzy probability function $\bar{P}$ is the representation of an uncertain expert opinion on the studied subject. The elements of $\bar{P}$ are symmetric triangular fuzzy numbers $\bar{P}\left(\left\{x_{i}\right\}\right)=\bar{\phi}_{i}=\left(a_{i} / u_{i} / b_{i}\right)$, for $1 \leq i \leq n$, where $a_{i}$ and $b_{i}$ are the pessimistic and optimistic estimates of $x_{i}$, respectively, and $u_{i}$ is the "the most likely" estimate.

When several experts provide these values, then $a_{i}$ and $b_{i}$ are obtained by calculating the variance of all the pessimistic and optimistic estimates, respectively, on the occurrence of $x_{i}$, and $u_{i}$ is the result of the arithmetic mean of all "most likely" estimates about the analyzed event [10, 22]. Hardly the obtained triangular fuzzy number is going to be symmetric, then one should transform this fuzzy number in order to obtain a new symmetric triangular fuzzy number denoted by

$$
\left(a_{i}^{*} / m_{i}^{*} / b_{i}^{*}\right),
$$

where

$$
m_{i}^{*}-a_{i}^{*}=\min \left(m_{i}-a_{i}, b_{i}-m_{i}\right),
$$

with $m_{i}^{*}-a_{i}^{*}=b_{i}^{*}-m_{i}^{*}$.

For $(X, \bar{\Phi})$ to represent a finite and discrete distribution of probabilities, it is necessary to make an arithmetic restriction, since the interval sum of the $\alpha$-cuts of all $\bar{\phi}_{i}$ hardly results in 1 [10]. Thus, for all $0 \leq \alpha \leq 1$, we choose a $e_{i}$ from each $\alpha$-cut $\bar{\phi}_{i}[\alpha]$ so that $\sum_{i=1}^{n} e_{i}=1$. So, instead of the interval sum of the $\alpha$-cuts necessarily results in 1 , we just need to obtain a single crisp value $e_{i}$ from each $\bar{\phi}_{i}[\alpha]$ in a way that the sum of all chosen $e_{i}$ results in 1 .

For that, consider the set of stochastic vectors

$$
E=\left\{\left(e_{1}, \ldots, e_{n}\right) \in[0,1]^{n} \mid \sum_{i=1}^{n} e_{i}=1\right\}
$$

the set

$$
\mathcal{A}_{\bar{\Phi}}^{\alpha}=\bar{\phi}_{1}[\alpha] \times \ldots \times \bar{\phi}_{n}[\alpha],
$$

which is the cartesian product of the elements of $\bar{\Phi}$, represented by $\alpha$-cuts. The set

$$
\mathcal{S}_{\bar{\Phi}}^{\alpha}=\mathcal{A}_{\bar{\Phi}}^{\alpha} \cap E
$$

is the domain of the functions that characterize the intended arithmetic restriction. Consider a subset $A \subseteq X$ and let

$$
I_{A}^{X}=\left\{i \in\{1, \ldots, n\} \mid x_{i} \in A\right\}
$$

be the set of indexes of the elements of $A$ related to the indexes of the set $X=\left\{x_{1}, \ldots, x_{n}\right\} .{ }^{3}$ For

\footnotetext{
${ }^{3}$ When the set of indexes of the elements of $X$ can be understood by context, then we denote the set of indexes from $A \subseteq X$ simply as $I_{A}$.
} 
$0 \leq \alpha \leq 1$, the function

$$
f_{\bar{\Phi}, \alpha}^{A}: \mathcal{S}_{\bar{\Phi}}^{\alpha} \rightarrow[0,1]
$$

given by

$$
f_{\bar{\Phi}, \alpha}^{A}\left(e_{1}, \ldots, e_{n}\right) \mapsto \sum_{i \in I_{A}} e_{i}
$$

defines the desired arithmetic restriction. Then, the fuzzy probability of a subset $A \subseteq X$ is obtained through its $\alpha$-cuts:

$$
\bar{P}(A)[\alpha]=\left\{f_{\bar{\Phi}, \alpha}^{A}(e) \mid e=\left(e_{1}, \ldots, e_{n}\right) \in \mathcal{S}_{\bar{\Phi}}^{\alpha}\right\} .
$$

Lemma 2 The set $\mathcal{S}_{\bar{\Phi}}^{\alpha}$ is formed by connected, closed and bounded intervals.

Proof. The proof is immediate, as $\mathcal{S}_{\bar{\Phi}}^{\alpha}$ is formed by the intersection of $E$ with $\mathcal{A}_{\bar{\Phi}}^{\alpha}$, which is formed by connected, closed and bounded intervals.

Proposition $3 \bar{P}(A)$ is a symmetric triangular fuzzy number.

Proof. It is immediate that the function $f_{\bar{\Phi}, \alpha}^{A}$ for some $I_{A}$ is continuous, and its domain $\mathcal{S}_{\bar{\Phi}}^{\alpha}$, as stated by the Lemma 2 , is formed by connected, closed and bounded intervals, signifying that the image of $f_{\bar{\Phi}, \alpha}^{A}$, given by $f_{\bar{\Phi}, \alpha}^{A}\left(\mathcal{S}_{\bar{\Phi}}^{\alpha}\right)=\bar{P}(A)[\alpha]$, is a connected, closed and bounded interval of real numbers. So, $\bar{P}(A)[\alpha]$ represents the $\alpha$-cuts of a fuzzy set $\bar{P}(A)$. Furthermore, $f_{\bar{\Phi}, 1}^{A}\left(\mathcal{S}_{\bar{\Phi}}^{1}\right)=\bar{P}(A)[1] \neq \emptyset$, as $\left(\phi_{1}, \ldots, \phi_{n}\right) \in \mathcal{S}_{\bar{\Phi}}^{1}$, so $\sum_{i \in I_{a}} \phi_{i} \in \bar{P}(A)[1]$, which means that $\bar{P}(A)$ is indeed a fuzzy number. As all $\bar{\phi}_{i} \in \bar{\Phi}$ are symmetric triangular fuzzy numbers, $\bar{\mu}_{\bar{\Phi}}^{r}$ will also be one, as none of the operations to calculate the fuzzy probability alters the form of the resulting fuzzy number [9].

Considering $A$ and $B$ as subsets of $X, \overline{0}=$ $(0 / 0 / 0)$ and $\overline{1}=(1 / 1 / 1)$, and using the ADorder [21], we have the following properties: $[12,13]$

a) $A \cap B=\emptyset \Rightarrow \bar{P}(A)+\bar{P}(B) \bar{\Sigma} \bar{P}(A \cup B)$.

b) $A \subseteq B \Rightarrow \bar{P}(A) \bar{\Sigma} \bar{P}(B)$.

c) $\forall A: \overline{0} \bar{\Sigma} \bar{P}(A) \overline{\leq} \overline{1}$;

d) $\bar{P}(\emptyset)=\overline{0} \wedge \bar{P}(X)=\overline{1}$;

e) $\bar{P}(A)+\bar{P}\left(A^{\prime}\right) \overline{\leq} \overline{1}$;

f) $A \cap B \neq \emptyset \Rightarrow \bar{P}(A)+\bar{P}(B)-\bar{P}(A \cap B) \bar{\Sigma} \bar{P}(A \cup B)$.

Finally, we show how to define the fuzzy mean $\bar{\mu}_{\bar{\Phi}}^{r}$ through the multiplication of each $\bar{\Phi}=\left\{\bar{\phi}_{1}, \ldots, \bar{\phi}_{n}\right\}$ by the real values in $r=\left(r_{1}, \ldots, r_{n}\right) \in \mathbb{R}^{n}$. To this end, we introduce the function

$$
g_{\bar{\Phi}, \alpha}^{r}: \mathcal{S}_{\bar{\Phi}}^{\alpha} \rightarrow \mathbb{R}
$$

defined by

$$
g_{\bar{\Phi}, \alpha}^{r}\left(e_{1}, \ldots, e_{n}\right)=\sum_{i=1}^{n} e_{i} \cdot r_{i}
$$

for $0 \leq \alpha \leq 1$, which ensures that the arithmetic restriction is respected. The fuzzy mean for a $r=$ $\left(r_{1}, \ldots, r_{n}\right) \in \mathbb{R}^{n}$ is defined through its $\alpha$-cuts, for $0 \leq \alpha \leq 1$ :

$$
\bar{\mu}_{\bar{\Phi}}^{r}[\alpha]=\left\{g_{\bar{\Phi}, \alpha}^{r}(e) \mid e=\left(e_{1}, \ldots, e_{n}\right) \in \mathcal{S}_{\bar{\Phi}}^{\alpha}\right\} .
$$

Proposition $4 \bar{\mu}_{\bar{\Phi}}^{r}$ is a triangular symmetric fuzzy number.

Proof. It is immediate that the function $g_{\bar{\Phi}, \alpha}^{r}$, for some $r=\left(r_{1}, \ldots, r_{n}\right) \in \mathbb{R}^{n}$, is continuous, and that its domain $\mathcal{S}_{\bar{\Phi}}^{\alpha}$, as stated in the Lemma 2, is constituted by connected, closed and bounded intervals, which implies that the image of $g_{\bar{\Phi}, \alpha}^{r}$, given by $g_{\bar{\Phi}, \alpha}\left(\mathcal{S}_{\bar{\Phi}}^{\alpha}\right)=\bar{\mu}_{\bar{\Phi}}^{r}[\alpha]$, is a closed and bounded interval of real numbers. So, $\bar{\mu}_{\bar{\Phi}}^{r}[\alpha]$ represents the $\alpha$-cuts of a fuzzy set $\bar{\mu}_{\bar{\Phi}}^{r}$. Furthermore, $g_{\bar{\Phi}, 1}^{r}\left(\mathcal{S}_{\bar{\Phi}}^{1}\right)=\bar{\mu}_{\bar{\Phi}}^{r}[1] \neq \emptyset$, as $\left(\phi_{1}, \ldots, \phi_{n}\right) \in \mathcal{S}_{\bar{\Phi}}^{1}$, and then $\phi_{1} \cdot r_{1}+\ldots+\phi_{n} \cdot r_{n} \in$ $\bar{\mu}_{\bar{\Phi}}^{r}[1]$, making $\bar{\mu}_{\bar{\Phi}}^{r}$ a fuzzy number. As all $\bar{\phi}_{i} \in \bar{\Phi}$ are symmetric triangular fuzzy numbers, $\bar{\mu}_{\bar{\Phi}}^{r}$ will also be one, as none of the operations to calculate the fuzzy mean alters the form of the resulting fuzzy number [9].

\section{Interval Fuzzy Probabilities}

We adopt an anologous approach for fuzzy probabilities in order to define interval fuzzy probabilities. Let $X=\left\{x_{1}, \ldots, x_{n}\right\}$ be a finite set and $P: \wp(X) \rightarrow[0,1]$ a probability function defined for all subsets of $X$, for $P\left(\left\{x_{j}\right\}\right)=\phi_{j}, 1 \leq j \leq n$, $0 \leq \phi_{j} \leq 1$ and $\sum_{i=1}^{n} \phi_{j}=1$, characterizing a discrete and finite probability distribution $\Phi=$ $\left\{\phi_{1}, \ldots, \phi_{n}\right\}$.

Consider that some of these $\phi_{j}$ represent imprecise values, in a way that even its fuzzy modeling is not trivial. Thus, we use interval fuzzy numbers substituting each $\phi_{j}$ for $\hat{\phi}_{j}$, constituting a new set $\hat{\Phi}$. Then, we have a interval fuzzy probability function $\hat{P}$, in a way that the elements of $\hat{\Phi}$ are represented by symmetric triangular interval fuzzy number $\hat{P}\left(x_{1}\right)=\hat{\phi}_{j}=\left(\bar{\phi}_{j}, \bar{\phi}_{j}\right)$, for $1 \leq j \leq n$, where $\bar{\phi}_{j_{i}}$ represents the fuzzy modeling with the lowest imprecision, and $\bar{\phi}_{j}$ represents the fuzzy modeling with the highest imprecision, according to the experts' opinions.

For $(X, \hat{\Phi})$ to represent a finite and discrete probability function, we define an arithmetic restriction. For $0 \leq \alpha_{1} \leq \alpha_{2} \leq 1$, we choose, from the $\left[\alpha_{1}, \alpha_{2}\right]$ cuts of each $\hat{\phi}_{j}$, denoted by $\hat{\phi}_{j}\left[\alpha_{1}, \alpha_{2}\right]$, an $e_{j}$ such that $\sum_{j=1}^{n} e_{j}=1$. Consider the set of stochastic vectors

$$
E=\left\{\left(e_{1}, \ldots, e_{n}\right) \in[0,1]^{n} \mid \sum_{j=1}^{n} e_{j}=1\right\},
$$

the set

$$
\mathcal{A}_{\hat{\Phi}}^{\alpha_{1}, \alpha_{2}}=\hat{\phi}_{1}\left[\alpha_{1}, \alpha_{2}\right] \times \ldots \times \hat{\phi}_{n}\left[\alpha_{1}, \alpha_{2}\right]
$$


of the cartesian product of the $\left[\alpha_{1}, \alpha_{2}\right]$-cuts of the elements of $\hat{\Phi}$, and

$$
\mathcal{S}_{\hat{\Phi}}^{\alpha_{1}, \alpha_{2}}=\mathcal{A}_{\hat{\Phi}}^{\alpha_{1}, \alpha_{2}} \cap E,
$$

which is the domain of the function that characterizes the arithmetic restriction. In order to guarantee that $\mathcal{S}_{\hat{\Phi}}^{\alpha_{1}, \alpha_{2}} \neq \emptyset$ is necessary to impose the restriction that 1 belong to $\sum_{j=1}^{n} \hat{\phi}_{j}\left[\alpha_{1}, \alpha_{2}\right]$.

Let $A \subseteq X$ be the index set of the elements of $A$, related to the indexes ${ }^{4}$ from $X$, defined by

$$
J_{A}^{X}=\left\{j \in\{1, \ldots, n\} \mid x_{j} \in A\right\} .
$$

The function $f_{\hat{\Phi},\left[\alpha_{1}, \alpha_{2}\right]}^{A}: \mathcal{S}_{\hat{\Phi}}^{\alpha_{1}, \alpha_{2}} \rightarrow[0,1]$, which characterizes the arithmetic restriction, is defined by:

$$
f_{\hat{\Phi},\left[\alpha_{1}, \alpha_{2}\right]}^{A}\left(e_{1}, \ldots, e_{n}\right)=\sum_{j \in J_{A}} e_{j},
$$

where $0 \leq \alpha_{1} \leq \alpha_{2} \leq 1$. The fuzzy probability of a subset $A \subseteq X$ is defined through its $\left[\alpha_{1}, \alpha_{2}\right]$-cuts:

$$
\hat{P}(A)\left[\alpha_{1}, \alpha_{2}\right]=\left\{f_{\hat{\Phi},\left[\alpha_{1}, \alpha_{2}\right]}^{A}(e) \mid e \in \mathcal{S}_{\hat{\Phi}}^{\alpha_{1}, \alpha_{2}}\right\} .
$$

Lemma 5 The set $\mathcal{S}_{\hat{\Phi}}^{\alpha_{1}, \alpha_{2}}$ is formed by connected, closed and bounded intervals.

Proof. The proof is immediate since the intersection of connected, closed and bounded intervals results in connected, closed and bounded interval.

Proposition $6 \hat{P}(A)$ is a symmetric triangular interval fuzzy number.

Proof. It is immediate that the function $f_{\hat{\Phi}, \alpha}^{A}$ is continuous and its domain $\mathcal{S}_{\hat{\Phi}}^{\alpha_{1}, \alpha_{2}}$, by Lemma 5 , is formed by connected, closed and bounded intervals, which implies that the image of $f_{\hat{\Phi},\left[\alpha_{1}, \alpha_{2}\right]}^{A}$, given by $f_{\hat{\Phi},\left[\alpha_{1}, \alpha_{2}\right]}^{A}\left(\mathcal{S}_{\hat{\Phi}}^{\alpha_{1}, \alpha_{2}}\right)=\hat{P}(A)\left[\alpha_{1}, \alpha_{2}\right]$, is a closed and bounded interval of real numbers. Thus, $\hat{P}(A)\left[\alpha_{1}, \alpha_{2}\right]$ is the $\left[\alpha_{1}, \alpha_{2}\right]$-cuts of a interval fuzzy set $\hat{P}(A)$. Furthermore, $f_{\hat{\Phi},[1,1]}^{A}\left(\mathcal{S}_{\hat{\Phi}}^{1,1}\right)=$ $\hat{P}(A)[1,1] \neq \emptyset$, as $\left(\phi_{1}, \ldots, \phi_{n}\right) \in \mathcal{S}_{\hat{\Phi}}^{1,1}$, so $\sum_{j \in J_{A}} \phi_{j} \in \hat{P}(A)[1,1]$, meaning that $\hat{P}(A)$ is indeed an interval fuzzy number. As all $\hat{\phi}_{i} \in \hat{\Phi}$ are symmetric triangular interval fuzzy numbers, also is $\hat{P}(A)[9]$.

Proposition 7 The interval fuzzy probability $\hat{P}(A)$ may be obtained through the calculation of its fuzzy generator probabilities $\bar{P}(A)_{i}$ and $\bar{P}(A)_{s}$, as:

$$
\hat{P}(A)\left[\alpha_{1}, \alpha_{2}\right]=\bar{P}(A)_{i}\left[\alpha_{1}\right] \cap \bar{P}(A)_{s}\left[\alpha_{2}\right],
$$

for $0 \leq \alpha_{1} \leq \alpha_{2} \leq 1$.

\footnotetext{
${ }^{4}$ When the set of indexes from $X$ can be understood by context, we denote the subset $A \subseteq X$ simply as $J_{A}$.
}

Proof. The proof is immediate, since $\hat{P}(A)=$ $\left(\bar{P}(A)_{i}, \bar{P}(A)_{s}\right)$, for $0 \leq \alpha_{1} \leq \alpha_{2} \leq 1$.

Observe that, for each $j$, one can denote $\hat{\phi}_{j}=$ $\left(\bar{\phi}_{j}, \bar{\phi}_{j_{s}}\right)$. Then, we can calculate the fuzzy probability $\bar{P}(A)_{i}\left[\alpha_{1}\right]$ through all $\bar{\phi}_{j}$, and the fuzzy probability $\bar{P}(A)_{s}\left[\alpha_{2}\right]$ through all $\bar{\phi}_{j_{s}}$. Once these fuzzy probabilities have been calculated, we only need to do the intersection of $\bar{P}(A)_{i}\left[\alpha_{1}\right]$ and $\bar{P}(A)_{s}\left[\alpha_{2}\right]$ to obtain $\hat{P}(A)\left[\alpha_{1}, \alpha_{2}\right]$, as given in the Eq. (15). So, we obtain the representation of interval fuzzy probabilities through their fuzzy generator probabilities as follows:

$$
\hat{P}(A)=\left(\bar{P}(A)_{i}, \bar{P}(A)_{s}\right) .
$$

The advantage in utilizing this kind of representation is that all the properties of fuzzy probabilities presented in the Sect. 3 are valid for interval fuzzy probabilities, which are calculated through the fuzzy generator probabilities and the order relation for interval fuzzy numbers based on the ADorder. Consider $\hat{0}=(\overline{0}, \overline{0}), \hat{1}=(\overline{1}, \overline{1})$ and $A, B \subseteq X$. We have the following properties:

a) $A \cap B=\emptyset \Rightarrow \hat{P}(A)+\hat{P}(B) \hat{\leq} \hat{P}(A \cup B)$.

Proof. We will show that

$\hat{P}(A)\left[\alpha_{1}, \alpha_{2}\right]+\hat{P}(B)\left[\alpha_{1}, \alpha_{2}\right] \supseteq \hat{P}(A \cup B)\left[\alpha_{1}, \alpha_{2}\right]$,

which means that $\hat{P}(A)+\hat{P}(B) \hat{\leq} \hat{P}(A \cup B)$, as stated by the interval AD-order. The Eq. (17) may be rewritten as follows:

$$
\begin{array}{r}
\left(\bar{P}(A)_{i}\left[\alpha_{1}\right] \cap \bar{P}(A)_{s}\left[\alpha_{2}\right]\right)+ \\
\left(\bar{P}(B)_{i}\left[\alpha_{1}\right] \cap \bar{P}(B)_{s}\left[\alpha_{2}\right]\right) \supseteq \\
\bar{P}(A \cup B)_{i}\left[\alpha_{1}\right] \cap \bar{P}(A \cup B)_{s}\left[\alpha_{2}\right] .
\end{array}
$$

Assuming that

$$
\begin{aligned}
& \bar{P}(A)_{i}\left[\alpha_{1}\right]+\bar{P}(B)_{i}\left[\alpha_{1}\right] \supseteq \bar{P}(A \cup B)_{i}\left[\alpha_{1}\right], \\
& \bar{P}(A)_{s}\left[\alpha_{2}\right]+\bar{P}(B)_{s}\left[\alpha_{2}\right] \supseteq \bar{P}(A \cup B)_{s}\left[\alpha_{2}\right],
\end{aligned}
$$

it follows that so the Eq. (18) is verified. The equations (19) and (20) are both true, as we are dealing with the same case discussed in the Sect. 3 for fuzzy probabilities of two disjoint sets.

b) $A \subseteq B \Rightarrow \hat{P}(A) \hat{\leq} \hat{P}(B)$.

Proof. One may observe that $A \subseteq$ $B \Rightarrow I_{A} \subseteq I_{B}$. So, considering $\hat{P}(A)\left[\alpha_{1}, \alpha_{2}\right]=\left[a_{1}(\alpha), a_{2}(\alpha)\right]$ and $\hat{P}(B)\left[\alpha_{1}, \alpha_{2}\right]=\left[b_{1}(\alpha), b_{2}(\alpha)\right]$, we always have $a_{1}(\alpha) \leq b_{1}(\alpha) \wedge a_{2}(\alpha) \leq b_{2}(\alpha)$, with $0 \leq \alpha \leq 1$, meaning that $\hat{P}(A) \hat{\leq} \hat{P}(B)$, as stated by the interval $\mathrm{AD}$ - order.

c) $\forall A: \hat{0} \hat{\leq} \hat{P}(A) \hat{\leq} \hat{1}$;

d) $\hat{P}(\emptyset)=\hat{0} \wedge \hat{P}(X)=\hat{1}$.

e) $\hat{P}(A)+\hat{P}\left(A^{\prime}\right) \hat{\leq} \hat{1}$. 
f) $A \cap B \neq \emptyset \Rightarrow \hat{P}(A)+\hat{P}(B)-\hat{P}(A \cap B) \hat{\leq} \hat{P}(A \cup B)$.

Proof. We have that

$$
\begin{array}{r}
\hat{P}(A)\left[\alpha_{1}, \alpha_{2}\right]+\hat{P}(B)\left[\alpha_{1}, \alpha_{2}\right]-\hat{P}(A \cap B)\left[\alpha_{1}, \alpha_{2}\right] \\
\supseteq \hat{P}(A \cup B)\left[\alpha_{1}, \alpha_{2}\right],
\end{array}
$$

for $0 \leq \alpha \leq 1$, which means by the interval AD-order that $\hat{P}(A)+\hat{P}(B)-\hat{P}(A \cap B) \hat{\leq} \hat{P}(A \cup$ $B)$. The Eq. (21) may be rewritten as:

$$
\begin{aligned}
\left(\bar{P}(A)_{i}\left[\alpha_{1}\right] \cap \bar{P}(A)_{s}\left[\alpha_{2}\right]\right)+\left(\bar{P}(B)_{1}\left[\alpha_{1}\right] \cap \bar{P}_{s}\left[\alpha_{2}\right]\right) & \\
-\left(\bar{P}(A \cap B)_{i}\left[\alpha_{1}\right] \cap \bar{P}(A \cap B)_{s}\left[\alpha_{2}\right]\right) & \supseteq \\
\bar{P}(A \cup B)_{i}\left[\alpha_{1}\right] \cap \bar{P}(A \cup B)_{s}\left[\alpha_{2}\right] . &
\end{aligned}
$$

Assuming that

$$
\begin{array}{r}
\bar{P}(A)_{i}\left[\alpha_{1}\right]+\bar{P}(B)_{i}\left[\alpha_{1}\right]-\bar{P}(A \cap B)_{i}\left[\alpha_{1}\right] \supseteq \\
\bar{P}(A \cup B)_{i}\left[\alpha_{1}\right],
\end{array}
$$

$$
\begin{array}{r}
\bar{P}(A)_{s}\left[\alpha_{2}\right]+\bar{P}(B)_{s}\left[\alpha_{2}\right]-\bar{P}(A \cap B)_{s}\left[\alpha_{2}\right] \supseteq \\
\bar{P}(A \cup B)_{s}\left[\alpha_{2}\right],
\end{array}
$$

we have that Eq. (21) is true. The equations (22) and (23) are also true, as they belong to the same case discussed in the Section 3 for fuzzy probabilities of two non-disjoint sets.

Example 1 We illustrate the property (a) through an example, showing that we may not obtain equality in that case. Let $A=\left\{x_{1}, x_{2}\right\}, B=\left\{x_{3}, x_{4}\right\}$ and $C=\left\{x_{5}\right\}$ be events (subsets) of the sample space $X=\left\{x_{1}, x_{2}, x_{3}, x_{4}, x_{5}\right\}$, so that $\sum_{i=1}^{5} P\left(x_{i}\right)=$ 1. All probabilities $P\left(x_{i}\right)$ are uncertain, except for $P\left(x_{5}\right)=0.2$. One way of represent this uncertainty is through fuzzy probabilities, so let $\bar{P}\left(x_{1}\right)=\bar{P}\left(x_{2}\right)=\bar{P}\left(x_{3}\right)=\bar{P}\left(x_{4}\right)=(0.19 / 0.2 / 0.21)$ and $P\left(x_{5}\right)=\bar{P}\left(x_{5}\right)=(0.2 / 0.2 / 0.2)$. Another way of modeling these fuzzy probabilities is admit further uncertainty, letting $\bar{P}\left(x_{1}\right)=\bar{P}\left(x_{2}\right)=$ $\bar{P}\left(x_{3}\right)=\bar{P}\left(x_{4}\right)=(0.18 / 0.2 / 0.22)$ and $\bar{P}\left(x_{5}\right)=$ $(0.2 / 0.2 / 0.2)$. If there is an impasse about which modeling is preferred, one may use interval fuzzy probabilities that cover all fuzzy modelings between the chosen fuzzy generator probabilities. Then, consider the following interval fuzzy modeling:

$$
\hat{P}\left(x_{i}\right)=((0.19 / 0.2 / 0.21),(0.18 / 0.2 / 0.22)),
$$

for each $i=1, \ldots, 4$ and

$$
\hat{P}\left(x_{5}\right)=((0.2 / 0.2 / 0.2),(0.2 / 0.2 / 0.2)) .
$$

With these interval fuzzy probabilities, $\hat{P}(A)=$ $\hat{P}(B)=((0.38 / 0.4 / 0.42),(0.36 / 0.4 / 0.44)) . \quad A s$ $\hat{P}(C)=\hat{P}\left(x_{5}\right)=((0.2 / 0.2 / 0.2),(0.2 / 0.2 / 0.2))=$ 0.2 , and $X=A \cup B \cup C$, the arithmetic restriction determines that $\hat{P}(A \cup B)=$ $((0.8 / 0.8 / 0.8),(0.8 / 0.8 / 0.8))=0.8$. The sum of $\hat{P}(A)$ and $\hat{P}(B)$ has no such restriction, and its obtained through the interval sum of the $\left[\alpha_{1}, \alpha_{2}\right]$-cuts of these interval fuzzy numbers. Thus, $\hat{P}(A)+\hat{P}(B)=((0.76 / 0.8 / 0.84),(0.72 / 0.8 / 0.88))$.
Through the interval AD-order we have that $\quad((0.76 / 0.8 / 0.84),(0.72 / 0.8 / 0.88)) \hat{<}$ $((0.8 / 0.8 / 0.8),(0.8 / 0.8 / 0.8))$, and so we conclude that $A \cap B=\emptyset$ and $\hat{P}(A)+\hat{P}(B) \hat{<} \hat{P}(A \cup B)$.

Now we introduce the calculation of the interval fuzzy mean $\hat{\mu}_{\hat{\Phi}}^{r}$, weighting the interval fuzzy probabilities in $\hat{\Phi}=\left\{\hat{\phi}_{1}, \ldots, \hat{\phi_{n}}\right\}$ by real values in $r=\left(r_{1}, \ldots, r_{n}\right) \in \mathbb{R}^{n}$. Similar to the calculation of the fuzzy mean, we define an auxiliary function $q_{\hat{\Phi},\left[\alpha_{1}, \alpha_{2}\right]}^{r}: \mathcal{S}_{\hat{\Phi}}^{\alpha_{1}, \alpha_{2}} \rightarrow \mathbb{R}$ as:

$$
q_{\hat{\Phi},\left[\alpha_{1}, \alpha_{2}\right]}^{r}\left(e_{1}, \ldots, e_{n}\right)=\sum_{j=1}^{n} e_{j} \cdot r_{j},
$$

with $0 \leq \alpha_{1} \leq \alpha_{2} \leq 1$. Thus, the interval fuzzy mean for a $r=\left(r_{1}, \ldots, r_{n}\right) \in \mathbb{R}^{n}$ is defined through its $\left[\alpha_{1}, \alpha_{2}\right]$-cuts as:

$$
\hat{\mu}_{\hat{\Phi}}^{r}\left[\alpha_{1}, \alpha_{2}\right]=\left\{q_{\hat{\Phi},\left[\alpha_{1}, \alpha_{2}\right]}^{r}(e) \mid e \in \mathcal{S}_{\hat{\Phi}}^{\alpha_{1}, \alpha_{2}}\right\}
$$

for $0 \leq \alpha_{1} \leq \alpha_{2} \leq 1$ and $q_{\hat{\Phi},\left[\alpha_{1}, \alpha_{2}\right]}^{r}: \mathcal{S}_{\hat{\Phi}}^{\alpha_{1}, \alpha_{2}} \rightarrow \mathbb{R}$ is defined by the Eq. (24).

Proposition $\mathbf{8} \hat{\mu}_{\hat{\Phi}}^{r}$ is a symmetric triangular interval fuzzy number.

Proof. It is immediate that the function $q_{\hat{\Phi},\left[\alpha_{1}, \alpha_{2}\right]}^{r}$ ' defined in Eq. (24) for some $r=\left(r_{1}, \ldots, r_{n}\right) \in \mathbb{R}^{n}$, is continuous and its domain $\mathcal{S}_{\hat{\Phi}}^{\alpha_{1}, \alpha_{2}}$, as stated by the Lemma 5 , is formed by connected, closed and bounded intervals, which implies that the image of $q_{\hat{\Phi},\left[\alpha_{1}, \alpha_{2}\right]}^{r}$, given by $q_{\hat{\Phi},\left[\alpha_{1}, \alpha_{2}\right]}\left(\mathcal{S}_{\hat{\Phi}}^{\alpha_{1}, \alpha_{2}}\right)=\hat{\mu}_{\hat{\Phi}}^{r}\left[\alpha_{1}, \alpha_{2}\right]$, is a closed and bounded interval of real numbers. Thus, $\hat{\mu}_{\hat{\Phi}}^{r}\left[\alpha_{1}, \alpha_{2}\right]$ represents the $\left[\alpha_{1}, \alpha_{2}\right]$-cuts of a interval fuzzy set $\hat{\mu}_{\hat{\Phi}}^{r}$. Furthermore, $q_{\hat{\Phi},[1,1]}^{r}\left(\mathcal{S}_{\hat{\Phi}}^{1,1}\right)=$ $\hat{\mu}_{\hat{\Phi}}^{r}[1,1] \neq \emptyset$, as $\left(\phi_{1}, \ldots, \phi_{n}\right) \in \mathcal{S}_{\hat{\Phi}}^{1,1}$, so $\phi_{1} \cdot r_{1}+\ldots+$ $\phi_{n} \cdot r_{n} \in \hat{\mu}_{\hat{\Phi}}^{r}[1,1]$, and then $\hat{\mu}_{\hat{\Phi}}^{r}$ is an interval fuzzy number. As all $\hat{\phi}_{i} \in \hat{\Phi}$ are triangular symmetric interval fuzzy numbers, $\hat{\mu}_{\hat{\Phi}}^{r}$ is, since we only do sums and scalar products [9].

Proposition 9 The interval fuzzy mean $\hat{\mu}_{\hat{\Phi}}^{r}$ may be obtained through the fuzzy generator means $\bar{\mu}_{\bar{\Phi}_{i}}^{r}$ and $\bar{\mu}_{\bar{\Phi}_{s}}^{r}$, for $0 \leq \alpha_{1} \leq \alpha_{2} \leq 1$, as:

$$
\hat{\mu}_{\hat{\Phi}}^{r}\left[\alpha_{1}, \alpha_{2}\right]=\bar{\mu}_{\bar{\Phi}_{i}}^{r}\left[\alpha_{1}\right] \cap \bar{\mu}_{\bar{\Phi}_{s}}^{r}\left[\alpha_{2}\right] .
$$

Proof.Similar to Proposition 7, Eq. (26) may be obtained considering $\hat{\mu}_{\hat{\Phi}}^{r}=\left(\bar{\mu}_{\bar{\Phi}_{i}}^{r}, \bar{\mu}_{\bar{\Phi}_{s}}^{r}\right)$ and $0 \leq \alpha_{1} \leq$ $\alpha_{2} \leq 1$.

As $\hat{\phi}_{j}=\left(\bar{\phi}_{j}, \bar{\phi}_{j_{s}}\right)$, we weigh the fuzzy probabilities in $\left\{\bar{\phi}_{1 i}, \ldots, \bar{\phi}_{n_{i}}\right\}$ by real values in $r=$ $\left(r_{1}, \ldots, r_{n}\right) \in \mathbb{R}^{n}$,and weight $\left\{\bar{\phi}_{1_{s}}, \ldots, \bar{\phi}_{n_{s}}\right\}$ by the same real values in $r=\left(r_{1}, \ldots, r_{n}\right) \in \mathbb{R}^{n}$, through the functions $g_{\bar{\Phi}_{i}, \alpha_{1}}^{r}$ and $g_{\bar{\Phi}_{s}, \alpha_{2}}^{r}$, defined by the Eq. (24).

So, we obtain the fuzzy generator means $\bar{\mu}_{\bar{\Phi}_{i}}^{r}\left[\alpha_{1}\right]$ and $\bar{\mu}_{\bar{\Phi}}^{r}\left[\alpha_{2}\right]$ (represented by their $\alpha$-cuts). By the intersection of these intervals, we obtain the interval fuzzy mean, represented by its $\left[\alpha_{1}, \alpha_{2}\right]$-cuts. 


\section{Conclusion}

The presented approach for interval fuzzy probabilities was shown as a viable way to represent imprecise probabilities with uncertainty in how the imprecision should be modeled. Coupled with the interval AD-order, this approach allows that certain properties of classical probability are maintained. This enables the expansion of problems where crisp probabilities are applied to versions with fuzzy or interval fuzzy probabilities.

It was observed that the interval fuzzy probabilities and interval fuzzy mean may be calculated using fuzzy generator probabilities, a simple alternative which takes advantage of the all the developed concepts for fuzzy probabilities.

Finally, for future work, we intend to use the presented approach to deal with the concept of interval fuzzy random variable and apply interval fuzzy probabilities in problems of social interaction based on agents, mainly in decision-making based on game theory $[12,24]$. Another potential application of this theory is the prediction of results of political elections or of games as made in [11].

Acknowledgments. This work was partially supported by the Brazilian funding agency CNPq (Conselho Nacional de Desenvolvimento Científico e Tecnológico), under the Proc. No. 481283/2013-7, 306970/2013-9, 307681/2012-2.

\section{References}

[1] D. Adamatti, G. P. Dimuro, and H. Coelho, editors. Interdisciplinary Applications of AgentBased Social Simulation and Modeling. IGI Global, Hershey, 2014.

[2] F. C. P. Santos, T. F. Rodrigues, H. Donancio, G. Dimuro, D. Adamatti, G. P. Dimuro, and E. Manuel Jerez. Analyzing the problem of the modeling of periodic normalized behaviors in multiagent-based simulation of social systems: The case of the San Jerónimo vegetable garden of Seville, Spain. In B. Kaminski and G. Koloch, editors, Advances in Social Simulation, volume 229 of Advances in Intelligent Systems and Computing, pages 61-72. Springer, Berlin, 2014.

[3] J. Sichman, A. Rocha Costa, D. Adamatti, G. Dimuro, and P. Bommel F. Buarque. An overview of social simulation research in Brazil. In Proceedings of 2012 Third Brazilian Workshop on Social Simulation (BWSS), pages 18 22. IEEE, Los Alamitos, 2012.

[4] W. Amaral and F. Gomide. A coevolutionary approach to solve fuzzy games. In R. Bello, R. Falcón, W. Pedrycz, and J. Kacprzyk, editors, Granular Computing: At the Junction of
Rough Sets and Fuzzy Sets, pages 121 - 130. Springer, Berlin, 2008.

[5] G. Parente Farias, G. Pereira Dimuro, G. Dimuro Peter, and E. de Manuel Jerez. A BDI-fuzzy agent model for exchanges of noneconomic services based on the social exchange theory. In Intelligent Systems (BRACIS), 2013 Brazilian Conference on, pages 26-32, Los Alamitos, 2013.

[6] H. Wu and M. Parlar. Games with incomplete information: A simplified exposition with inventory management applications. International Journal of Production Economics, 133(2):562 - 577, 2011.

[7] L. A. Zadeh. Fuzzy sets. Information and Control, 8(3):338-353, 1965.

[8] L. A. Zadeh. The concept of a linguistic variable and its application to approximate reasoning - I. Information Sciences, 8(3):199-249, 1975.

[9] G. P. Dimuro. On interval fuzzy numbers. In 2011 Workshop-School of Theoretical Computer Science (WEIT), pages 3-8, Los Alamitos, 2011. IEEE.

[10] J. J. Buckley. Fuzzy Probabilities: New Approach and Applications. Springer, Berlin, 2005.

[11] C. G. Costa, B. Bedregal, and A. D. Dória Neto. Atanassov's intuitionistic fuzzy probability and Markov chains. Knowledge-Based Systems, 43(0):52 - 62, 2013.

[12] T. C. Asmus, B. R. C. Bedregal, and G. P. Dimuro. On Interval Fuzzy Bayesian Games: Preliminary Results, chapter 24, pages 131136. World Scientific, Singapore, 2014.

[13] T. C. Asmus and G. P. Dimuro. On fuzzy probabilities in Bayesian games. In 2011 Workshop-School on Theoretical Computer Science, WEIT 2011, pages 25-31, Los Alamitos, CA, USA, 2011. IEEE Computer Society.

[14] B. C. Bedregal. On interval fuzzy negations. Fuzzy Sets and Systems, 161(17):2290-2313, 2010 .

[15] B. C. Bedregal, G. P. Dimuro, R. H. N. Santiago, and R. H. S. Reiser. On interval fuzzy S-implications. Information Sciences, 180(8):1373-1389, 2010.

[16] G. P. Dimuro, B. C. Bedregal, R. H. N. Santiago, and R. H. S. Reiser. Interval additive generators of interval t-norms and interval tconorms. Information Sciences, 181(18):3898 - 3916, 2011.

[17] B. C. Bedregal, G. P. Dimuro, and R. H. S. Reiser. An approach to interval-valued R-implications and automorphisms. In João Paulo Carvalho, Didier Dubois, Uzay Kaymak, and João Miguel da Costa Sousa, editors, Proceedings of the Joint 2009 International Fuzzy Systems Association World Congress and 2009 European Society of 
Fuzzy Logic and Technology Conference, IFSA/EUSFLAT, pages 1-6, 2009.

[18] B. R. C. Bedregal and R. H. N. Santiago. Some continuity notions for interval functions and representation. Computational \& Applied Mathematics, 32:435-446, 2013.

[19] R. E. Moore. Methods and Applications of Interval Analysis. SIAM, Philadelphia, 1979.

[20] R. H. N. Santiago, B. C. Bedregal, and B. M. Acióly. Formal aspects of correctness and optimality in interval computations. Formal Aspects of Computing, 18(2):231-243, 2006.

[21] T. C. Asmus and G. P. Dimuro. A total order for symmetric triangular (interval) fuzzy numbers. Mathware \& Soft Computing Magazine, 20(1):76-114, 2013.

[22] J. J. Buckley and E. Eslami. Uncertain probabilities I: the discrete case. Soft Computing - A Fusion of Foundations, Methodologies and Applications, 7(8):500-505, 2003.

[23] L. A. Zadeh. Fuzzy probabilities. Information Processing and Management, 20(3):363372, 1984.

[24] Y. Shoham and K. Leyton-Brown. Multiagent Systems: Algorithmic, Game-Theoretic, and Logical Foundations. Cambridge University Press, Cambridge, 2009. 